UCID- $\top 8681$

The Effects of Dimensionality and

Boundary Cond:tions on Themal Calculations

Lewis D. Thorson

Donald N. Montan

September 29, 1980

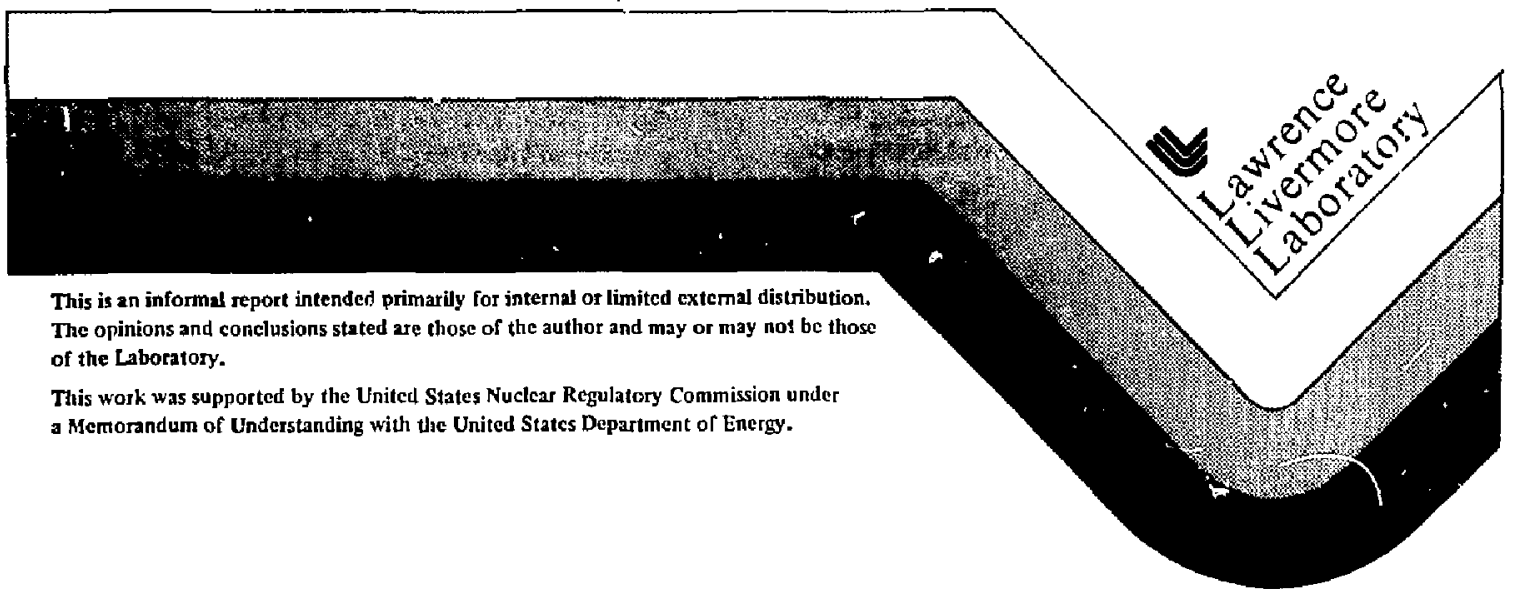




\section{IABLE OF CONTENTS}

age

List of Figures

Abstract

Introduction

Tise Physical Problem

The Required Analyt ic Solutions

Energy Conservation Doing Superposition

Results

Conclusions

v

1

1

2

3

4

6

References

iil 


\section{List of Figures}

Figure

1

2

3

4

5

6

7 $\underline{\text { Title }}$

$\underline{\text { Page }}$

Infinite Medium 9

Ground Surface as Perfect Insulator 10

Ground Surface is Isothermal 11

2-D Case, - Infinite Medium,

-- Surface = Insulator

2-D Case, - Infinite Medium,

-- Surface Isotherma]

3-D Case, - Real Power History,

-- Constant Power

2-D Case, - Real Power History,

-- Constant Power
12

13

14

11 
We consider several aspects of the thermal modeling of an underground nuclear waste repository. These are the long term temperature response due to different ground surface boundary conditions, the effects of treating the three-dimensional problem in 2-D, and the conseyuences of using a constant power load rather than decaying power typical of radioactive waste. Our approach is superposition in time and space of the appropriate analytic solutions.

\section{INTRODUCTION}

Thermal effects are an important aspect of the nuclear waste repository problem. The mecharical response in the rock mass and the flow of fluid around the repository are but two physical effects that are temperature dependent. Hence an accurate calculation of the heat load is an important issue.

A frequent simplification when doing numerical thermal modeling of the region araund a nuclear maste repository is to use a vertical two-dimensional grid. This reduces both the comple.ity and the memory requirements for the problem. The validity of this assumption is usualiy ignored and it is this that we seek to address with particular emphasis on the long term thermal history.

Secondly, we consider the effect of the ground surface boundary condition on the temperature distribution throughout the medium. It is well known that boundary conditions are of primary importance in determining the temperature distribution. To assess this effect a comparison was made between a repositury situated in an infinite medium and one where the ground surface was either a perfect insulator or isothermal. These conditions were chosen since they are the usual ones to start with when doing numerical modeling. For some types of problems the ground surface may have to be handled in a more "realistic" fastion. These questions are problem dependent and are not addressed here. These types of surface conditions are important on the other boundariec as well. The question of how large to make a numerical grid must also be considered. The results can be directly carried over to this situation. 
Lastly we considered the consequences of using a constant power load rather than decaying power typical of radioactive waste.

The problems considered were approached using the appropriate analytic solutions. This method was chosen for ease of solution and clarity of results.

\section{The Physical Problem}

We consider a ectangular repository $l \mathrm{~km}$ on a side situated $400 \mathrm{~m}$ below the ground surface. The surrounding medium has a thermal conductivity of $2 \mathrm{w} / \mathrm{m}-\mathrm{K}$ and a thermal diffusivity of $30 \mathrm{~m}^{2} /$ year. These values are typicai of some granites and basalts. The canisters were assumed to contain 10 year old fuel at a power level of $1 \mathrm{~kW}$. At the time of emplacement the total areal rower load was fixed at $100 \mathrm{kH} / \mathrm{acre}$. These values are typical of waste repositories and a surruunoing medium.

The power table below. from Raines, (1) was useu to give a typical power history for the calculations. The values used are for fuel from a pressurized water reactor. The table was normalized to the desired heat load.

\begin{tabular}{rr}
$\begin{array}{c}\text { Heat Generated } \\
\text { Years After } \\
\text { Discharge }\end{array}$ & $\begin{array}{c}\text { PWR } \\
\text { W/MTHM }\end{array}$ \\
\hline 0 & $0.1742 \mathrm{E}+07$ \\
1 & $0.1043 \mathrm{E}+05$ \\
2 & $0.5640 \mathrm{E}+04$ \\
3 & $0.3598 \mathrm{E}+04$ \\
4 & $0.2568 \mathrm{E}+04$ \\
5 & $0.2011 \mathrm{E}+04$ \\
6 & $0.1688 \mathrm{E}+04$ \\
7 & $0.1487 \mathrm{E}+04$ \\
8 & $0.1353 \mathrm{E}+04$ \\
9 & $0.1259 \mathrm{E}+04$ \\
10 & $0.1189 \mathrm{E}+04$ \\
20 & $0.8904 \mathrm{E}+03$ \\
30 & $0.7394 \mathrm{E}+03$ \\
40 & $0.6246 \mathrm{E}+03$ \\
50 & $0.5340 \mathrm{E}+03$ \\
100 & $0.2886 \mathrm{E}+03$ \\
200 & $0.1627 \mathrm{E}+03$ \\
400 & $0.1099 \mathrm{E}+03$ \\
600 & $0.8455 \mathrm{E}+02$ \\
1,000 & $0.5547 \mathrm{E}+12$ \\
10,000 & $0.1380 \mathrm{E}+02$ \\
30,000 & $0.5507 \mathrm{E}+01$ \\
100,000 & $0.1069 \mathrm{E}+01$ \\
1000,000 & $0.3919 \mathrm{E}+00$ \\
&
\end{tabular}




\section{The Required Analytic Solutions}

The required analytic solutions are readily available from Cars law and Jaeger. (2) They are used along with superposition and images to generate the desired results.

Consider the case of a vertical two dimensional problem. This implies an inf inite repository into the vertical plane. Since a repository will consist of a series of drifts the problem can be approximated by a series of line sources with the proper power alloted each to give the desired power load per unit length of the repositury. (It is assumed that the observation points are a sufficient distance away that the individual waste canisters appear as series of line sources.) We can then use the solution for a continuous line source

where

$$
T=\frac{q}{4 \pi k} E_{1}\left(\frac{r^{2}}{4 k t}\right)
$$

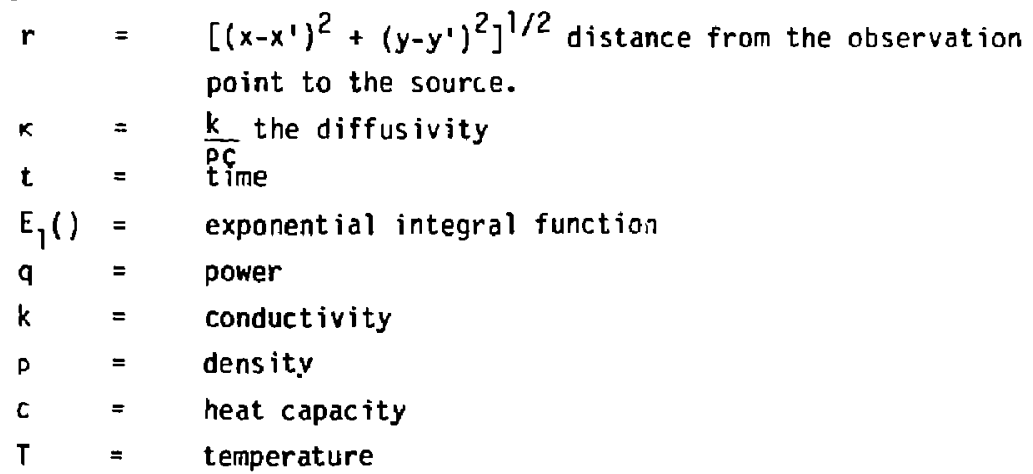

A three-dimensional repository requires a finite line source for each drift. While one can derive the temperature for a finite line formally it is difficult to do the required superposition in space and $t$ ime for the waste repository. Instead consider the solution for a continuuus point source solution:

$$
T=\frac{q}{4 \pi k r} \operatorname{erfc}\left(\frac{r}{\sqrt{4 k t}}\right)
$$

Where erfc() is the complementary error function and the other symbols are as before. Each drift can then be represented by many point sources. Superposition in space and time is used as before to model the repository. 
Energy Conservation Doing Superposition

In doing discrete time superposition, consideration must be given to properly determine the time to change power levels. In the case of a discrete power history, this time is well determined. If the power is a linear function of time, linear interpolation gives the desired result.

However a power table for mixed fission products falls off in a logarithmic fashion. In this case linear interpolation requires the use of small time increments, which proves to be costly for the time frames of interest. If linear interpolation is used with large time steps, energy is not conserved, and the temperatures are too high. To circumvent this situation we seek a $t$ ime, $t_{s}$, which will produce a histogram which canserves energy.

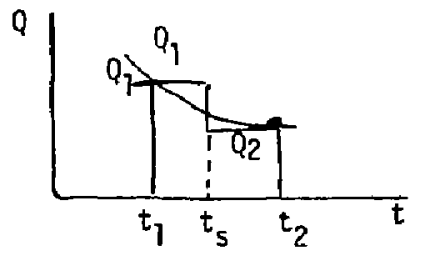

If the power at $t$ ime $t_{1}$ is $Q_{1}$ and at time $t_{2}$ is $Q_{2}$ we $c$ an calculate the total energy as

$$
E=\int_{t_{1}}^{t_{2}} u d t
$$

For an interpolation time, $t_{s}$, between $t_{1}$ and $t_{2}$, we split the integral in $t$ wo and get

$$
E=Q_{1}\left(t_{5}-t_{1}\right)+Q_{2}\left(t_{2}-t_{5}\right)
$$


Solving for $t_{s}$ gives

$$
t_{s}=\frac{E+\left(Q_{1} t_{1}-Q_{2}-t_{2}\right)}{a_{1}-Q_{2}}
$$

To this point the results are general. To apply them to a particular probiem requires a relatianship of $Q$ and time. For our ca.e it is appropriate to use a log power-log time relation, such as

$$
Q=a_{1}\left(\frac{t}{t_{1}}\right)^{\alpha}
$$

Then

$$
\begin{aligned}
E=\int_{t_{l}}^{t_{2}} 0 j t & =\frac{1}{a+1} \frac{Q_{1}}{t_{1}^{\alpha}}\left(t_{2}^{a+1}-t_{1}^{a+1}\right) \\
& =\frac{q_{1} t_{1}}{\alpha+1}\left[\left(\frac{t_{2}}{t_{1}}\right)^{a+1}-1 j\right.
\end{aligned}
$$

this is used in Eq. (4) to get

$$
t_{5}=t_{1}\left(\frac{a}{a+1}\right) \frac{1-\frac{a_{2}}{Q_{1}} \frac{t_{2}}{t_{1}}}{1-\frac{Q_{2}}{Q_{1}}}
$$

Eliminating a from the equation the desired :esult is

$$
t_{s}=t_{1} \frac{\ln \left(\frac{Q_{2}}{Q_{1}}\right)}{\ln \left(\frac{Q_{2}}{Q_{1}}\right)+\ln \left(\frac{t_{2}}{t_{1}}\right)} \frac{1-\frac{Q_{2}}{Q_{1}} \frac{t_{2}}{t_{1}}}{1-\frac{Q_{2}}{Q_{1}}}
$$

This then, is the time used to do the superposition. In this way a more accurate answer is obtained and the calculation runs faster since fewer time superpositions are needed. 
RESULTS

The results presented consist of graphs of temperature vs time at 4 positions; $100 \mathrm{~m}, 200 \mathrm{~m}, 300 \mathrm{~m}$ and $400 \mathrm{~m}$ (ground surface) abcve the mid-point of the repository. We include the cases of an infinite medium, and the ground surface as either a perfect insulator or isothermal.

The first set of figures shows comparisons of the finite and the infinite repositcry, i.e., the 3-D (solid line) vs 2-0 (dashed line) case. Figure 1 is the case where the repository is placed in an infinite medium. There is good agreement unt 1700 years when the inf inite repository temperatures begin to diverge. In Fig. 2 the ground surface is made a perfect insulator; aga in agreement is good up to 700 years. Away from the repository the temperature is higher and the overall gradient drops due to the insulating boundary. The case where the surface is isothermal is shown in Fig. 3 . Here the $400 \mathrm{~m}$ plot, the ground surface, is not shown since the temperature is always zero. The temperatures are lower and the gradients higher than the infinite media case.

Since the observation points are situated on the midline above the repository the times when the $2-D$ and $3-D$ results diverge and the subsequent differences, should show the least effect. At any other location these differences shouid be greater. It is through the fourier number, $\left(k t / l^{2}\right.$, being the distance to the point of concern) that one can determine when "significant" differences occur. The magnitude of the Fourier numbers, and hence the temperature difference, has to be determined for each problem based on the purpose of the calculation and the desired accuracy. The point in time where the 2-D and 3-D results diverge corresponds to a Fourier number which indicates that portions of the 2-D (infinite line) source beyond the extent of the 3-D (finite line) source have had a significant effect on the temperature history at the observation point. From this it should be clear that the 3-0 temperature will always be less than or equal to the 2-D results. These results show that if one is only interested in the first 500-700 years either solution will suffice. It should be remembered that this time frame is dependent on bath the physical properties of the medium and the location of observation points relative to the repository. 
We next investigated the effect of the surface boundary condition on the 2-D repository case. Here the solid line is always the case of the repository in an infinite medium. In Fig. 4 the dashes are for an insulated surface. Here the curves diverge at 400 years, and there is a shift in the neak in both time and temperature. There is also a large change in the temperature gradients. In Fig. 5 the dashes correspond to an isothermal ground surface. Again the plot for the $400 \mathrm{~m}$ point disappears. Now the divergence occurs at 700 years. As beiore these resuits are dependent on the medium parameters and the position of the observation points.

It should be quite clear that one needs to consider both the dimensionality of the problem and the ground surface boundary conditions. At "short" time and at the mid-point above a repository either solution can be used. But each new situation must be judged separately.

In each of the previous cases little difference can be seen in the temperature history. There is one error that can show up at very early time. This occurs if the power is held at a constant level, $1 \mathrm{~kW}$ per unit length, rather than following the correct power history. This case is included for completeness. For this purpose it is only necessary to show the results for an infinite medium. In Fig. 6 the solid line is the 3-D repository with the correct power history and the dashes show the case for constant power. It is clearly seen that after 50 years there is too much heat being put into the system. Fig. 7 shows the same result for the 2-D repository. The closer one gets to the repository the sooner this divergence would be seen. It should be clear that to run the power at a constant level is not appropriate.

\section{CONCLUSIONS}

From these calculations several conclusions can be drawn:

1) Careful attention must be paid to the appropriateness of using an infinite, i.e. 2-D geometry, to simulate an actual waste repository. This involves consideration of both the medium coefficients and the position of the observation point. The use of the faurier number, $k t / l^{2}$, determines when the simplification can be made. 
2) The ground surface boundary condition has a strong influence on both the temperature and its gradient. Every problem must be carefully analyzed to determine when the boundaries come into play and if that is of significance to the resulis. Again consideration of the Fourier number is indicated.

3) Using a constant power must he avoided. A real power table is required.

\section{Ref erences:}

1. "Technical Progress Report for the Quarter July 1 - September 30, 1979, office of Nuclear Waste Isolation, Battelle Memorial Institute, ONWI-9(4).

2. H. S. Carslaw and J. C. Jaeger, "Conduction of Heat in Solids," Second Ed., Oxford at the Clarendon Press, 1959. 




FIG. 1 INF/MITE MEDIUM

DISTANCES ABOVE REPOSITORY, GROUND SURFACE AT $400 \mathrm{M}$ 


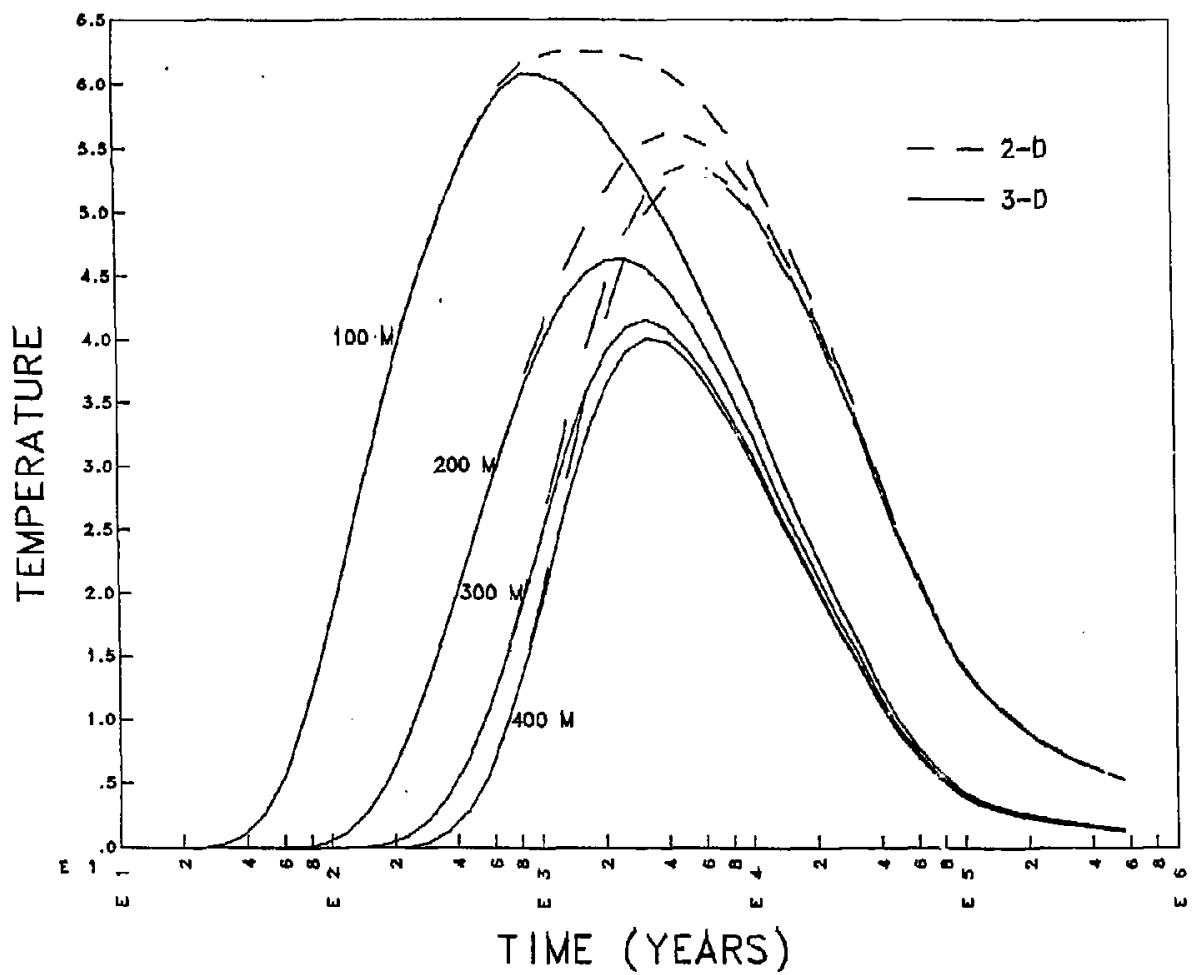

F IG. 2 GROUND SURFACE AS PERFECT INSULATOR

DISTANCES ABOVE REPOSITORY, GROUND SURFACE AT 4OOM 


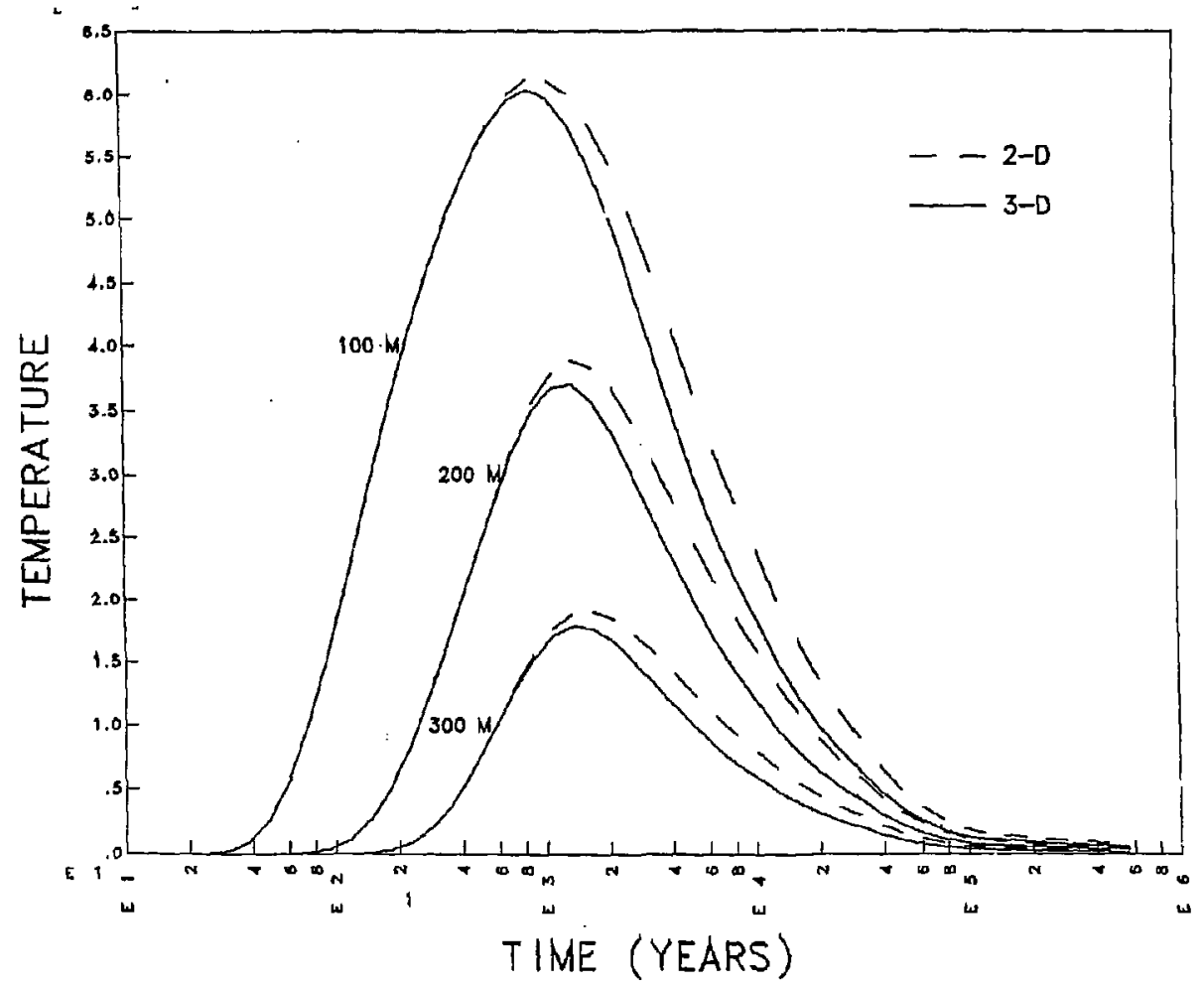

FIG, 3 ground SURFace is isothermal

DISTANCES ABOVE REPOSITORY, GROUND SURFACE AT $400 \mathrm{M}$ 


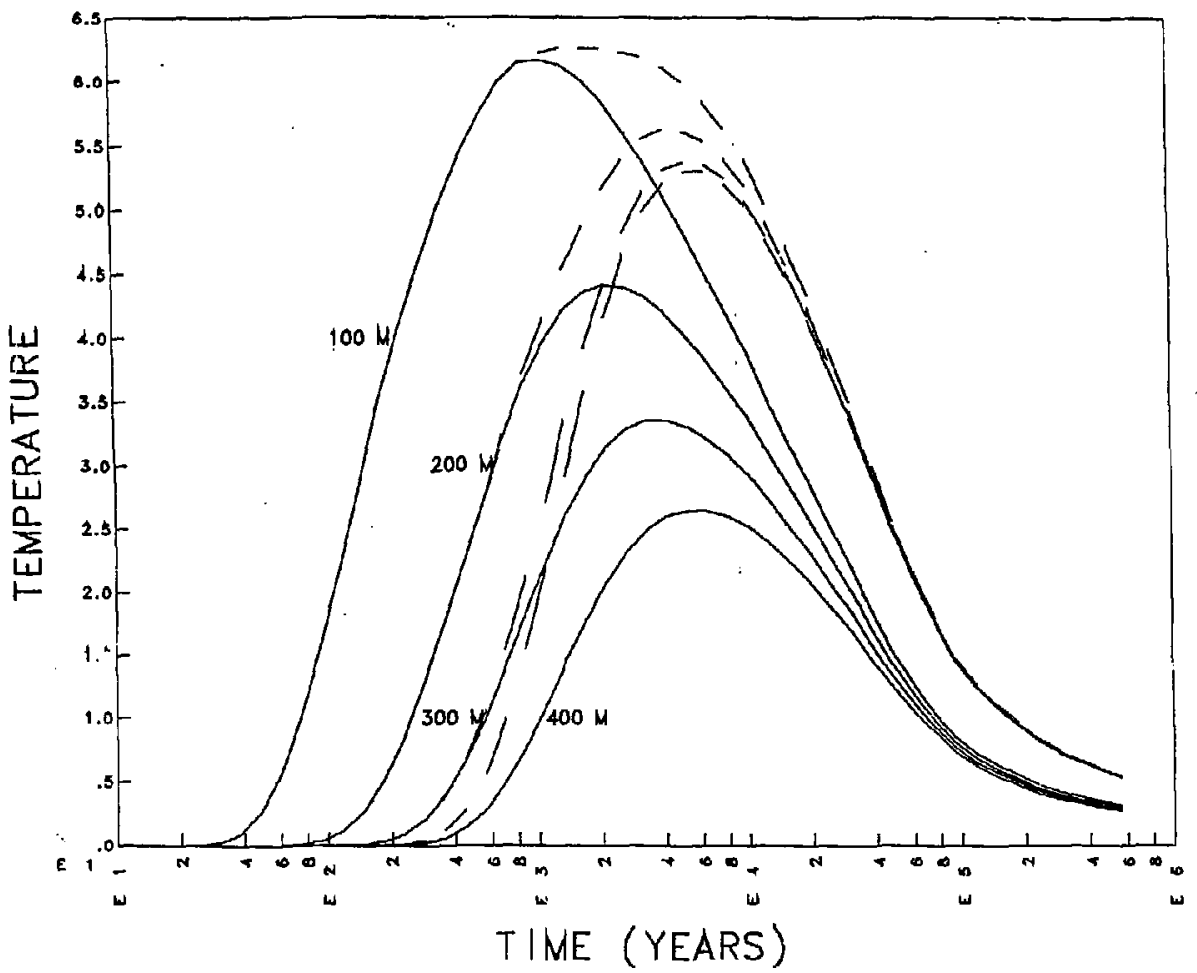

FIG. 4 2-D CASE: - INFINITE MEDIUM, - - SURFACE = INSULATOR DISTANCES ABOVE REPOSITORY, GROUND SURFACE AT 4OOM 


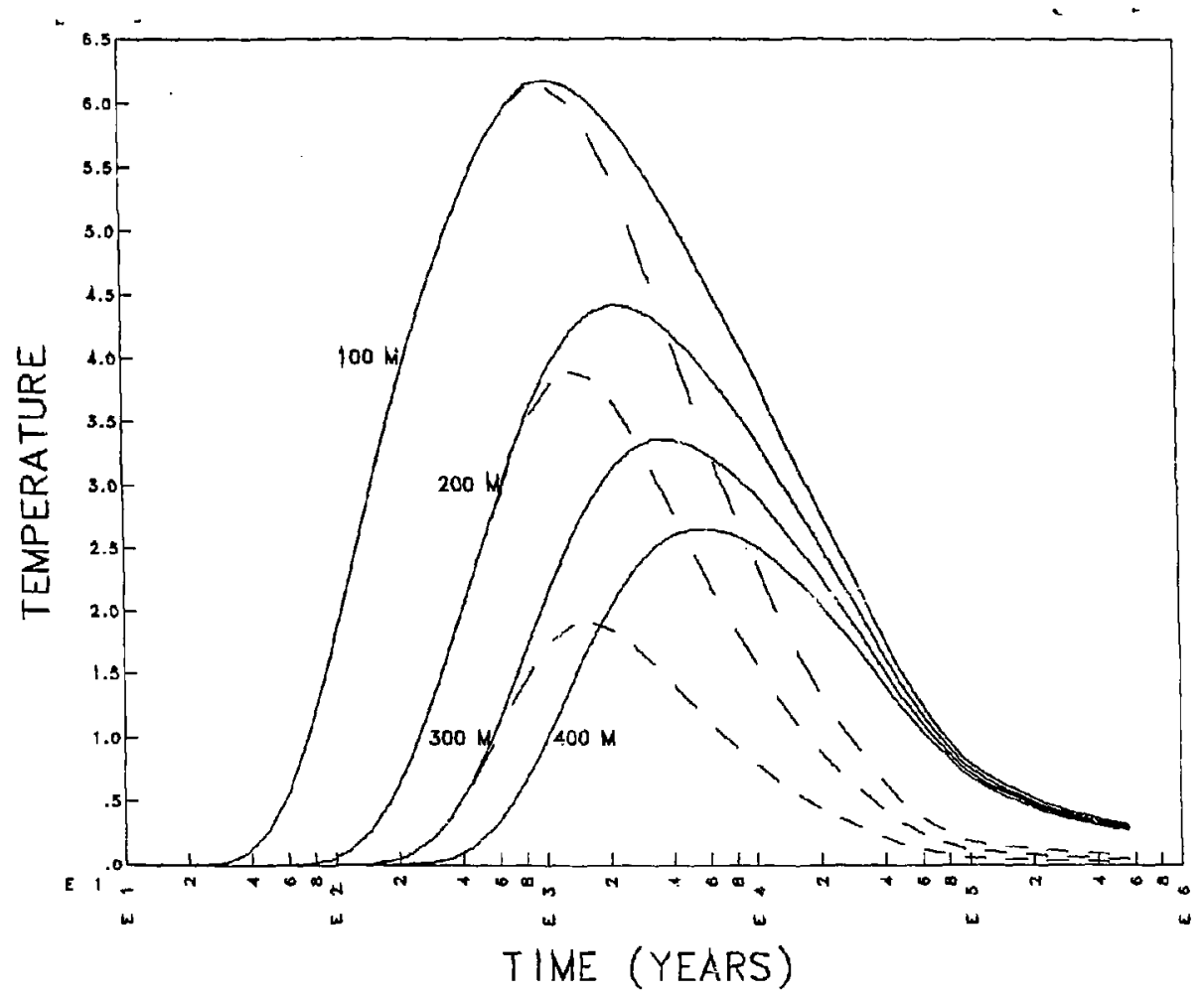

FIG, 5 2-D CASE: — INFINITE MEOIUH, - - SURFACE ISOTHERMAL DISTANCES ABOVE REPOSITORY. GROUND SURFACE AT 4OON 




FIG. 6 3-D CASE; - REAL POWER HISTORY, - - CONSTANT POWER DISTANCES ABOVE REPOSITORY. GROUND SURFACE AT 4OOM 


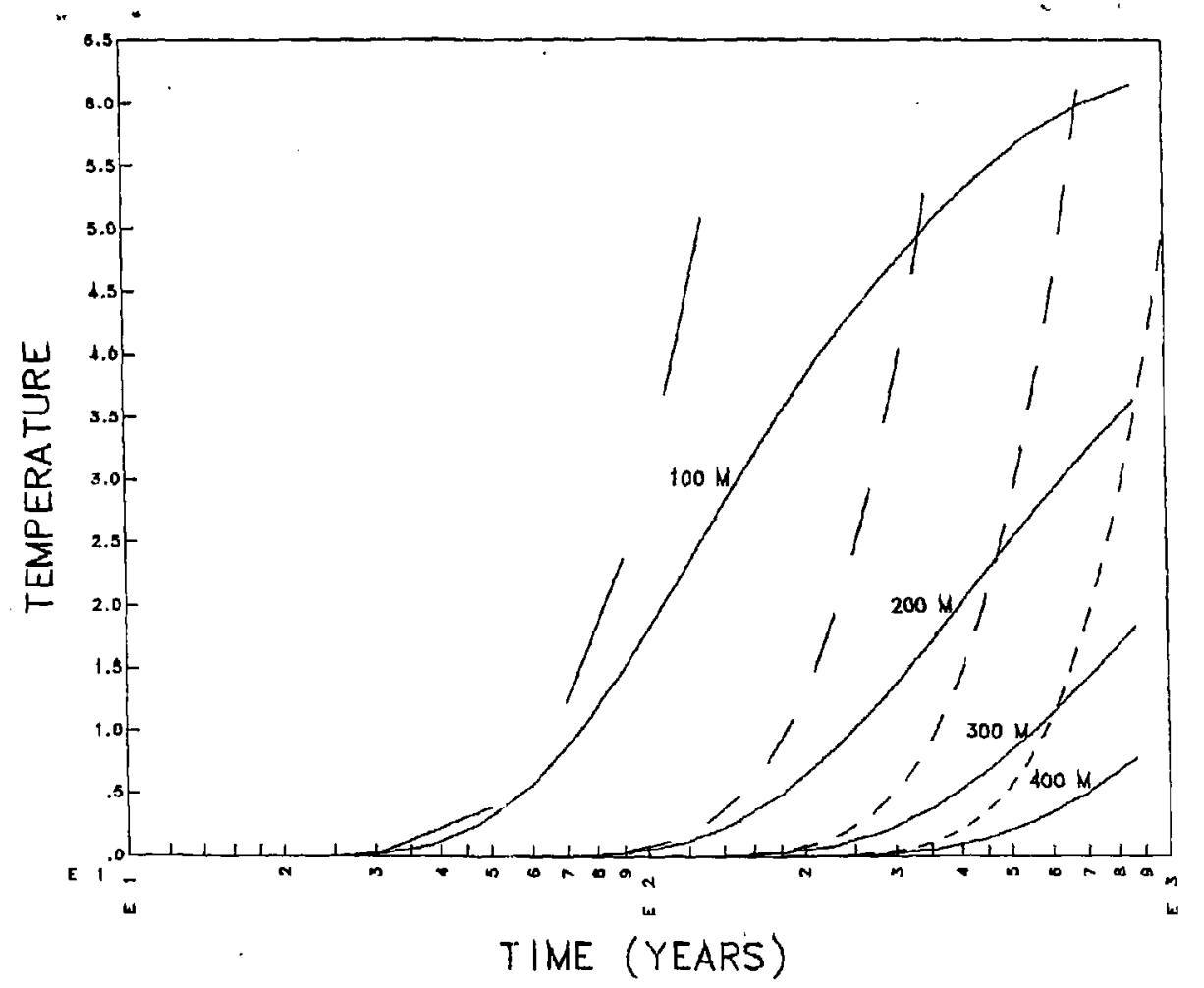

FIG. 7 2-D CASE; — REAL POWER HISTORY, - - CONSTANT POWER UISTANCES ABOVE REPOSI TORY. GROUND SURFACE AT HOOM 